\title{
Preparation, characterization, and application of a lipophilic coated exfoliated Egyptian blue for near- infrared luminescent latent fingermark detection
}

\author{
Sorour Shahbazi ${ }^{a}$, John V. Goodpaster ${ }^{b}$, Gregory D. Smithc, Thomas Beckera, \\ Simon W. Lewis ${ }^{\mathrm{a},}$ \\ ${ }^{a}$ Curtin Institute of Functional Molecules and Interfaces and School of Molecular and Life Sciences, Curtin \\ University, GPO Box U1987, Perth, Western Australia 6845, Australia \\ ${ }^{b}$ Department of Chemistry and Chemical Biology, Forensic and Investigative Sciences Program, Indiana \\ University Purdue University Indianapolis (IUPUI), 402 North Blackford Street, LD 326, Indianapolis, IN, \\ 46202, USA
}

${ }^{\mathrm{c}}$ Indianapolis Museum of Art at Newfields, 4000 Michigan Road, Indianapolis, IN, 46208, USA

*Author for correspondence: s.lewis@curtin.edu.au

\begin{abstract}
A simple technique is described to create nanoscale near-infrared luminescent Egyptian Blue pigment particles with lipophilic surfaces for latent fingermark detection. An exfoliated Egyptian blue powder coated with cetrimonium bromide was prepared using a simple, one-pot process. Particle size and surface coatings were characterized using scanning electron and atomic force microscopies, dynamic light scattering, and infrared and luminescence spectroscopies. This new fingermark dusting powder presented more contrast compared to uncoated Egyptian blue of comparable particle size as well as commercial visible light fluorescent (Blitz Red) and near infrared luminescent (fpNatural1) powders in the detection of aged fingermarks on model surfaces. The coated Egyptian blue pigment presents a cost-effective fingermark dusting powder that reveals high contrast latent fingermarks with simple, inexpensive photography equipment.
\end{abstract}

Keywords: Forensic science; Latent fingermarks; NIR luminescence; Egyptian blue; Nanostructured materials

This is the author's manuscript of the work published in final edited form as: 


\section{Introduction}

Powder dusting is the most frequently used method for latent fingermark detection on non-porous substrates [1]. An important consideration when selecting a fingerprint dusting powder is the nature of the substrate, with photoluminescent powders being advantageous when dealing with dark, multicoloured or patterned substrates. Luminescence increases the contrast between latent fingermarks ridges and the underlying substrate by moving the means of detection outside of the ambient illumination $[\underline{2}, \underline{3}]$.

A wide range of photoluminescent powders are commercially available, with most exhibiting fluorescence within the visible part of the spectrum $(400-700 \mathrm{~nm})$ when irradiated in the ultraviolet region. However, there are many potentially interfering substances that also fluoresce within this region and could contribute to background interference, including printing inks, bodily fluids, and optical brighteners [4-6]. Nearinfrared (NIR) region (700-1000 nm) luminescent materials have potential to improve detectability of fingermarks since very few substrates luminesce strongly in this region [6-9]. Despite this advantage, only a limited number of NIR luminescent powders have been reported [10-13].

A recent addition to these previously reported powders is the ancient pigment Egyptian blue $\left(\mathrm{CaCuSi}_{4} \mathrm{O}_{10}\right)[\underline{14}, \underline{15}]$. It has been used for decorative purposes since ancient times and is still commercially available as an artist's pigment today. Egyptian blue is made of several layers of calcium copper tetrasilicate [16, 17] which strongly luminesce at $910 \mathrm{~nm}$ when exited with visible illumination, especially at around $630 \mathrm{~nm}$ [18-21]. Conservation scientists have utilized this property to identify the pigment's use on 
antiquities via NIR photography since about 1990 [르. Egyptian blue is a very durable pigment that is largely insensitive to oxygen, $\mathrm{pH}$ and temperature, although its chemical reactivity is not completely understood [14, 23]. The pigment is also inexpensive and non-toxic, which makes the powder very suitable for latent fingermark detection on a wide range of substrates without background interference.

Egyptian blue was successfully applied for detecting latent fingermarks on diverse nonporous multicoloured substrates $[\underline{14}, \underline{15}, \underline{24}]$. It was found that the large particle size of commercial pigment powder (approximately $50 \mu \mathrm{m}$ ) caused weak adhesion between the colourant particles and the fingermark secretions, and therefore, the particle size had to be reduced through milling. The particle size of commercial pigment material was reduced to approximately $5 \mu \mathrm{m}$ after 30 minutes of micronising with corundum grinding pellets [14]. However, gradual surface wear of the corundum pellets was reported, which leads to significant corundum contamination over long grinding periods and a resulting dilution of the luminescent material [14]. Contemporaneous with this work, King et al. reported a similar approach using Egyptian blue as a fingerprint powder, although utilising a different excitation band in the NIR $(780 \mathrm{~nm})$ to avoid issues of interferences with the NIR luminescent dyes that appear to be used for United Kingdom polymer banknotes [15].

Johnson-McDaniel et al. [23] have reported a method in which the multilayered structure of Egyptian blue crystals is delaminated under very simple experimental conditions. In this method, pigment was stirred in hot water $\left(\sim 80^{\circ} \mathrm{C}\right)$ to exfoliate the copper silicate layers and produce Egyptian blue nanosheets. They also studied the physical and photophysical characteristics of the nanosheets for their potential 
applications in materials science. The strong luminescence is retained even in this nanoscale form.

One major issue related to the powder dusting method is a potential lack of selectivity and sensitivity owing to the inherently weak adhesion between the inorganic particles and the organic secretion residues. This method is also less effective for aged latent fingermarks that have lost moisture through evaporation or experienced a change of the lipid components due to evaporation to form harder, less sticky waxes $[\underline{25}, \underline{26}]$. In order to increase sensitivity, selectivity and adhesion, lipid-modified powders have been developed to enhance latent fingermark detection $[\underline{27}, \underline{28}]$. Cetrimonium bromide (CTAB, cetyltrimethylammonium bromide, $\mathrm{C}_{16} \mathrm{H}_{33} \mathrm{~N}^{+}\left(\mathrm{CH}_{3}\right)_{3} \mathrm{Br}^{-}$) is a cationic lipophilic surfactant that could interact electrostatically with the negative surface charge of Egyptian blue particles, thereby rendering them more attracted to latent fingermark residue.

Here we present an approach to decrease the size of Egyptian blue particles for fingermark detection using a straightforward and inexpensive approach, without the disadvantages of mechanical milling. A facile, one pot coating technique was applied for surface modification of exfoliated Egyptian blue with the CTAB surfactant. Properties of uncoated and lipophilically-modified powders were determined using advanced materials characterization techniques including electron and atomic force microscopy as well as luminescence and infrared (IR) spectroscopy. The modified powder was evaluated for its ability to reveal latent fingermarks on a range of substrates including highly patterned, Australian polymer banknotes. 


\section{Materials and Methods}

\subsection{Materials}

A commercially available Egyptian blue pigment (Kremer Pigmente Inc., Germany) was used in this study. CTAB (Sigma-Aldrich Pty. Ltd., 99 \%), palmitic acid (Sigma-Aldrich Pty. Ltd., $\geq 99 \%$ ), and n-dodecylamine acetate (Optimum Technology, Australia) were employed as lipophilic coatings. Blitz Red® (Criminal Research Products, LLC) and fpNatural1 (Pathtech, Foster+Freeman, Australia) powders were used as commercially available fluorescent fingermark powders for comparison. Ceramic tiles (Bunnings Warehouse, Australia), frosted microscope glass slides (Thermofisher scientific, Labserv, Australia), glass petri dishes, and new Australian banknotes (5 AUD obtained from a bank, http://banknotes.rba.gov.au/banknote-features/\#-new-five) issued only a few months prior to the research were used as substrates.

\subsection{Decreasing Egyptian blue pigment size}

Exfoliated Egyptian blue powder was prepared using a modified version of the method of Johnson-McDaniel et al. [223]. Three grams of Egyptian blue pigment and $90 \mathrm{ml}$ of deionised water were stirred using a glass-coated magnet in a round-bottom flask for 11 days at $85{ }^{\circ} \mathrm{C}$. The exfoliated Egyptian blue particles were isolated using a centrifuge (5000 rpm for $3 \mathrm{~min}$ ), and the supernatant was decanted. Exfoliated Egyptian blue particles were left to dry overnight in air or in an oven (All-Lab Scientific, ZRD-A5055) at $30{ }^{\circ} \mathrm{C}$

To coat the exfoliated Egyptian blue particles, $1 \mathrm{~g}$ of CTAB powder was added to the mixture of Egyptian blue and water inside the round-bottom flask before stirring. After 
coating, excess $\mathrm{CTAB}$ was removed by 3 times rinsing the exfoliated Egyptian blue particles with deionised water and centrifuging as described above.

For comparison, micronised Egyptian blue pigment was prepared as described by Errington et al. [14] using a McCrone micronising mill; however, agate pellets were used instead of corundum to minimize possible contamination.

Prior to their use in testing, all of the resultant Egyptian blue powders were stored in an oven at $30{ }^{\circ} \mathrm{C}$. The powders were found to agglomerate, possibly due to humidity. Storing them in the oven at $30^{\circ} \mathrm{C}$ before fingermark powder dusting almost solved the problem.

In a preliminary experiment, Egyptian blue particles were surface modified with some lipophilic coatings including CTAB, palmitic acid (PA), and n-dodecylamine acetate (nDA). A half gram of the coating agent was stirred with Egyptian blue powder in $40 \mathrm{ml}$ of methanol for 1 day.

\subsection{Electron microscopy}

A ZEISS EVO 40 XVP scanning electron microscope (SEM) was used for investigating the morphology and size of the Egyptian blue particles. Data was collected from secondary electron emissions using a variable pressure secondary electron (VPSE) detector. Acceleration voltages of 15 and $20 \mathrm{kV}$, spot size $300 \mathrm{~nm}$, and working distances (WD) 9.0 and $9.5 \mathrm{~mm}$ were applied for imaging.

For sample preparation, particles were dispersed in deionised water and sonicated for approximately 2 minutes, then particles were spin coated on a glass slide at $3000 \mathrm{rpm}$ for 2 minutes. Afterward, samples were carbon coated to increase conductivity. 


\subsection{Atomic force microscopy}

Phase and height imaging of Egyptian Blue particles was carried out using a WITec atomic force microscope (AFM) in intermittent mode, $\mathrm{f}_{0}=320 \mathrm{kHz}, \mathrm{k}=42 \mathrm{~N} / \mathrm{m}$, using a NCHV-A probe (Al coating on cantilever backside). The experiment was conducted under ambient conditions. AFM imaging of both the coated and uncoated particles was performed under the same instrumental settings and conditions.

For sample preparation, particles were dispersed in deionised water and sonicated for about 1 minute before being spun coated onto mica substrates at $1500 \mathrm{rpm}$ for 1.5 minutes.

\subsection{Infrared spectroscopy}

A Thermo Scientific Nicolet iS50 Fourier transform infrared (FTIR) spectrometer with an attenuated total reflection (ATR) module and deuterated triglycine sulfate (DTGS) detector was used to study chemical bonding within the prepared Egyptian blue powders. The spectrometer was equipped with a pressure arm that was used to maintain a constant $27 \mathrm{~kg}$ contact force between the sample and the diamond sampling crystal. Spectra were recorded over the range of $400-4000 \mathrm{~cm}^{-1}$ with 64 accumulated scans per sample, and $4 \mathrm{~cm}^{-1}$ spectral resolution. Samples were analysed as dried powders, and no special sample preparation was needed.

\subsection{Luminescence spectroscopy}

Emission spectra of the prepared Egyptian Blue powders were collected using an Edinburgh FLS980 spectrometer equipped with $450 \mathrm{~W}$ ozone-free xenon arc lamp and a Hamamatsu R5509-42 detector. The spectral range of this spectrometer is from the deep ultraviolet (UV) at $200 \mathrm{~nm}$ to the IR at $1700 \mathrm{~nm}$. Nuclear magnetic resonance 
(NMR) glass sample tubes (5 mm in diameter, Sigma-Aldrich Pty., USA) were used for holding to hold the powdered inside the sample chamber.

\subsection{Dynamic light scattering (DLS)}

A Malvern Zetasizer Nano ZS instrument was applied for measuring the zeta potential of the prepared Egyptian blue powders using a Capillary Zeta Cell (DTS 1060). Low concentrated suspensions of the powders in Milli-Q water were sonicated for less than 30 seconds. For each suspension, the surface charges of three subsamples were measured ( 5 runs per subsample and a total of 15 runs per suspension).

\subsection{Collection of latent fingermarks specimens}

Charged (enhanced) and uncharged (natural) latent fingermarks were collected from eight donors (4 males and 4 females aged between 25-50 years) on tiles, glass slides or new 5 AUD banknotes. For charging the fingermarks, donors were asked to rub the tips of their fingers across the nose and forehead to transfer sebaceous components to the fingermark secretion. For collecting the uncharged latent fingermarks, donors were asked not to wash their hands or touch chemicals at least 30 minutes before fingermark donation; then, fingermarks samples were collected by touching the substrates and holding their fingers on them for approximately 4 seconds. All fingertips were rubbed together before fingermark deposition to uniformly distribute skin secretions. Fingermark depletion series to assess the sensitivity of the method were prepared by asking donors to press the same fingers sequentially (up to 4 times) on the substrate to produce a series of increasingly weaker secretion residues. Split fingermarks were used in comparative testing. 
Three comparisons of Egyptian blue fingermark dusting powders were made between micronised and uncoated exfoliated (2-day old fingermarks), uncoated and CTAB coated exfoliated (2-day old fingermarks), as well as micronised and coated exfoliated (2-day and 15-day old fingermarks) Egyptian blue. These trials were conducted by powder dusting a 4 depletions in each series of uncharged split fingermarks from 6 donors deposited on glass slides, giving a total of 192 impressions. To collect split fingermarks, two glass slides were attached side by side and three middle fingers were used for deposition. A second trial was performed to compare CTAB coated exfoliated Egyptian blue to the commercial fluorescent fingermark powder, Blitz Red. Twelve 2-day old uncharged split impressions from 6 donors were deposited on new 5 AUD banknotes as an example of a common multicoloured, patterned substrate. To study the applicability of the coated exfoliated Egyptian blue powder, a 15-day old 4 depletion series from 6 donors and 2-month aged uncharged fingermarks from 2 donors (a total of 52 impressions) were collected on different substrates including glass Petri dish, 5 AUD notes, and ceramic tiles. The International Fingerprint Research Group (IFRG) guidelines were followed in terms of the number of donors, collection of fingermarks, and the splitmark approach [29]. Substrates on which fingermarks were deposited were washed thoroughly with detergent and rinsed with acetone and deionised water several times before each deposition.

All fingermark samples were stored in an office environment away from sunlight without any light protection. The temperature $\left(20-23{ }^{\circ} \mathrm{C}\right)$ and relative humidity (2867\%) were monitored using a Digitech QP-6013 datalogger over the period of storage. 


\subsection{Latent fingermark powder dusting}

Various prepared Egyptian blue powders were applied using a camel hair brush (Model Master, 3/8", USA). Previous research has highlighted the importance of using a natural hair brush when working with Egyptian blue powders [14]. However, Blitz Red was applied using a single-use disposable fiberglass brush (TheBreeze, USA). A swirling movement of the brush was used when applying all powders.

\subsection{Photography}

A modified Canon EOS 40D camera was used for photography of the fingermarks dusted with Egyptian blue powders. As part of the modification, the camera's internal IR blocking filter, which normally reduces the natural sensitivity of CCD and CMOS sensors to IR radiation, was removed. The camera was equipped with a Canon EFS 18-55 mm lens and a RM90 IR long-pass filter, which cuts off transmission of radiation shorter than approximately $900 \mathrm{~nm}[\underline{30}]$.

A Nikon D300 camera with a $60 \mathrm{~mm}$ lems, fitted with a Schott OG550 1\% $529 \mathrm{~nm}$ orange filter was used for photography of the Blitz Red $₫$ developed fingermarks. The Nikon camera was connected to a desktop computer running Camera Control Pro software (version 2.0.0) to use the live view and remote control functions of the camera.

Both cameras were installed on a Firenze mini repero stand in a darkened room for the respective imaging. The aperture, ISO, and shutter speed of each photo were chosen to enhance contrast and visibility of the dusted fingermarks and are provided under each figure.

Developed fingermarks on various substrates were illuminated either with a white light LED square array (Camera Electronics, North Perth) or a forensic light source (Polilight 
PL500, Rofine, Australia). The Polilight source used excitation wavelengths of 590 and $505 \mathrm{~nm}$ for Egyptian blue and Blitz Red powders, respectively.

The images of fingermarks were processed and adjusted in brightness using Adobe Photoshop CC (version 14.1.2×64) to make ridge details more visible. However, the same modifications were applied on the fingermarks in all comparison studies.

\section{Results and discussion}

\subsection{Production of surface modified exfoliated Egyptian Blue}

Calcium copper tetrasilicate layers of Egyptian blue were broken and exfoliated by stirring in hot water at $85{ }^{\circ} \mathrm{C}$ for 11 days. While delamination into smaller particles predictably changed the colour of the powder from bright blue to pale blue, the exfoliated nanosheets remained strongly luminescent in the NIR region when illuminated with visible light (Figure 1). Figure 2 shows SEM and AFM images of commercial Egyptian blue pigment and the exfoliated Egyptian blue particles at different magnifications. As illustrated in Figure 2c-2f, the resultant powder was a mixture of exfoliated small particles (Figure 2c and 2d) and nanosheets (Figure 2e and 2f). The nanosheet dimensions were less than $200 \mathrm{~nm}$ in length and less than $5 \mathrm{~nm}$ in thickness. However, there were still intact Egyptian blue particles with the same size as the unexfoliated particles (approximately $10 \mu \mathrm{m}$ ). 

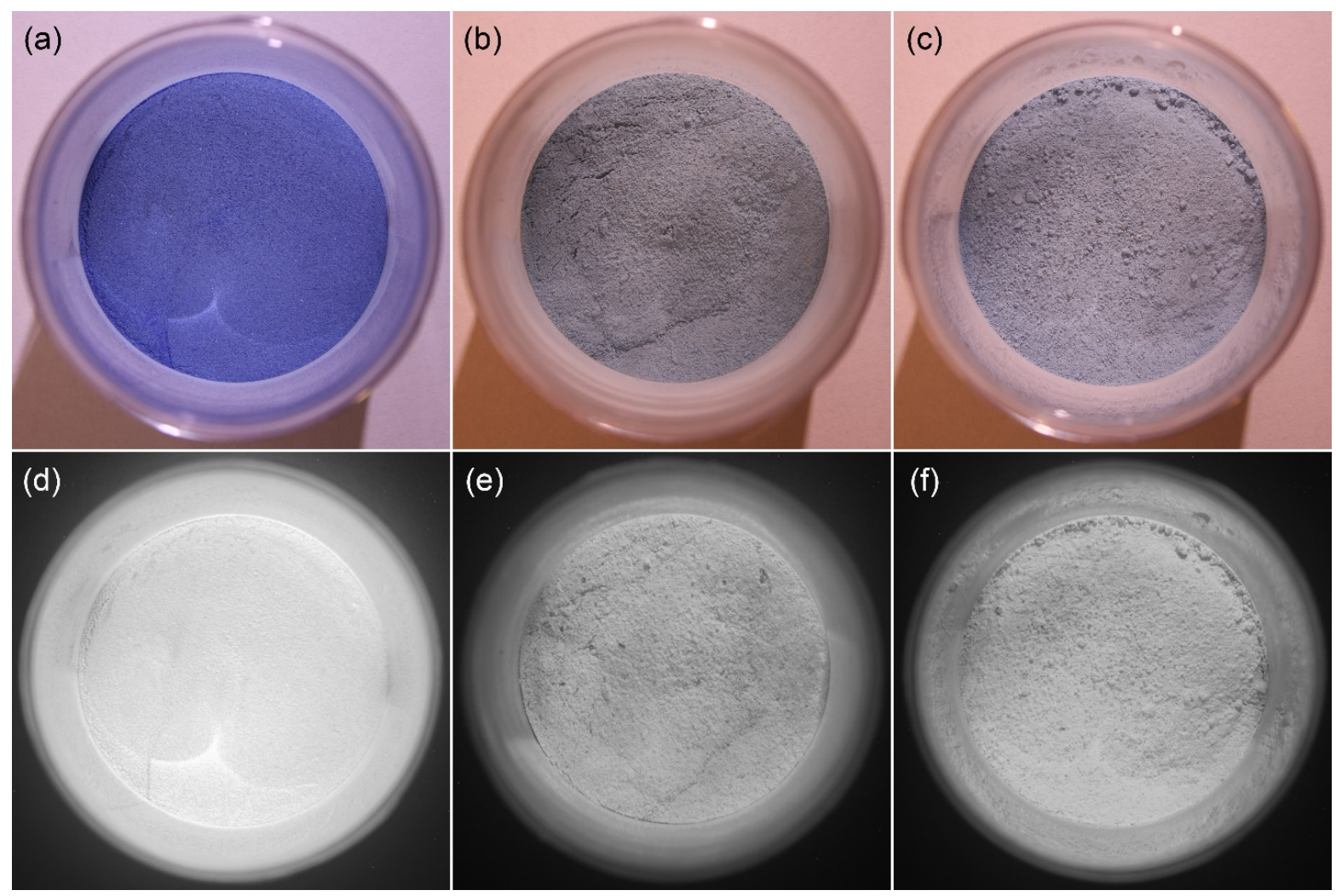

Figure 1 Photographs of Egyptian blue pigment ( $a$ and d) exfoliated Egyptian blue ( $b$ and e), and CTAB coated exfoliated Egyptian blue (c and f) using a Canon D40 camera under white light illumination (top row, aperture f/9, shutter speed1/30 s, ISO 200) and under Photoluminescent mode (bottom row, excitation with a Polilight PL 500 at $590 \mathrm{~nm}$ excitation and viewed through an IR long-pass filter, aperture f/9, shutter speed 2s, ISO 200). 


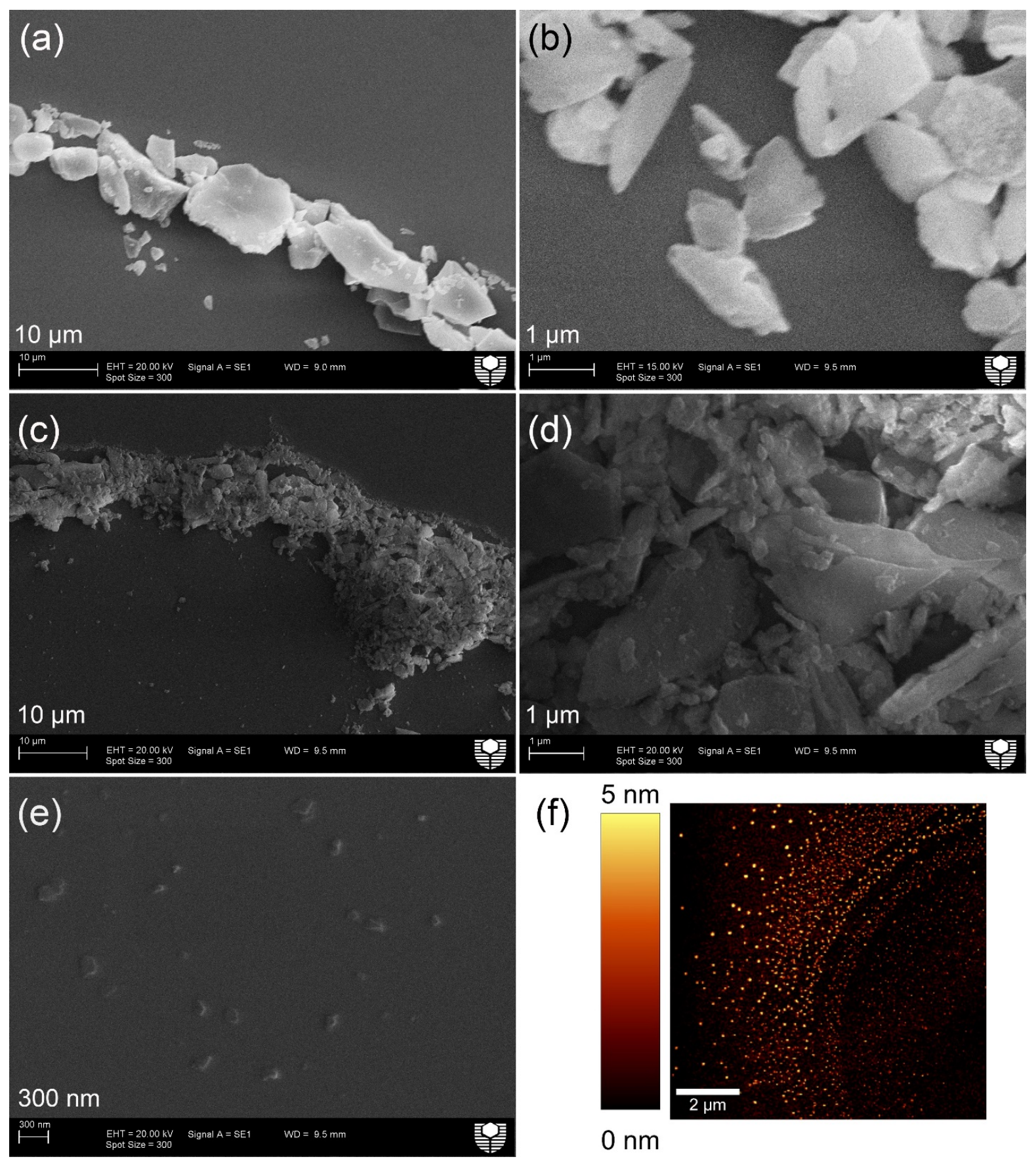

Figure 2 SEM images at different magnifications of Egyptian blue pigment ( $a$ and b), exfoliated Egyptian blue particles ( $c$ and d), exfoliated Egyptian blue nanosheets (e), and an AFM image of exfoliated Egyptian blue nanosheets $(f)$.

Preliminary investigations explored the potential of coating Egyptian blue particles with various lipophilic surfactants including CTAB, PA, and n-DA. These surfactants have long carbon chains that increase their lipophilic properties, potentially increasing their interaction with lipidic or waxy residues common to fingermarks. Figure 3 reveals the luminescence typical of both uncharged and charged latent fingermarks powdered with 
coated Egyptian blue pigment. Increased adhesion of Egyptian blue particles to fingermarks was observed for all coated particles compared to uncoated ones. It should be considered that contrast of uncoated Egyptian blue powder could be increased with higher exposure time, however, coated Egyptian blue powders had much higher luminescent intensities so the photography conditions to keep them in high contrast suppress the luminescence of the uncoated Egyptian blue. Although n-DA showed the strongest luminescence of the surface modified particles dusted onto charged fingerprints, the image lacked high level ridge detail. However, the CTAB coated particles yielded noticeably improved results not only on charged latent fingermarks, but also on uncharged ones. Therefore, subsequent experiments focused solely on CTAB as a surfactant for surface modification of Egyptian blue pigment.

Figure 4 shows AFM images of topography and phase signal for CTAB coated and uncoated exfoliated Egyptian blue nanosheets. Comparing Figure 4a and Figure 4b, coated particles with approximately the same size as uncoated ones appear slightly brighter than the uncoated ones indicating a larger thickness of coated exfoliated particles. Also, Figure 4c and Figure $4 \mathrm{~d}$ show phase imaging of CTAB coated and uncoated particles respectively. Less contrast was observed for the CTAB coated particles indicating a different interaction between AFM probe and the respective particles. This altered interaction can be attributed to the surface coating of the exfoliated Egyptian nanosheets which is softer than the Egyptian blue particles [31].

An attempt was made to estimate the Egyptian blue surface charge using DLS. Based on 15 replicate measurements, the average of surface charge of the Egyptian blue and uncoated exfoliated Egyptian blue powders were respectively $-30.8 \pm 1.2 \mathrm{mV}$ and 21.6 \pm 0.8 . However, the zeta potential measurements of the CTAB coated exfoliated 
Egyptian blue powder showed a positive surface charge (an average of $+23.2 \pm 1.9 \mathrm{mV}$ ) in comparison with the negative surface charge of the uncoated particles. The negative charge of the uncoated particles could be a result of exposed silicate and/or copper oxide ions in the structure. Since CTAB is a cationic surfactant, electrostatic interactions between the surfactant and the negative surface charge of the Egyptian blue pigment is assumed to be the coating mechanism. 


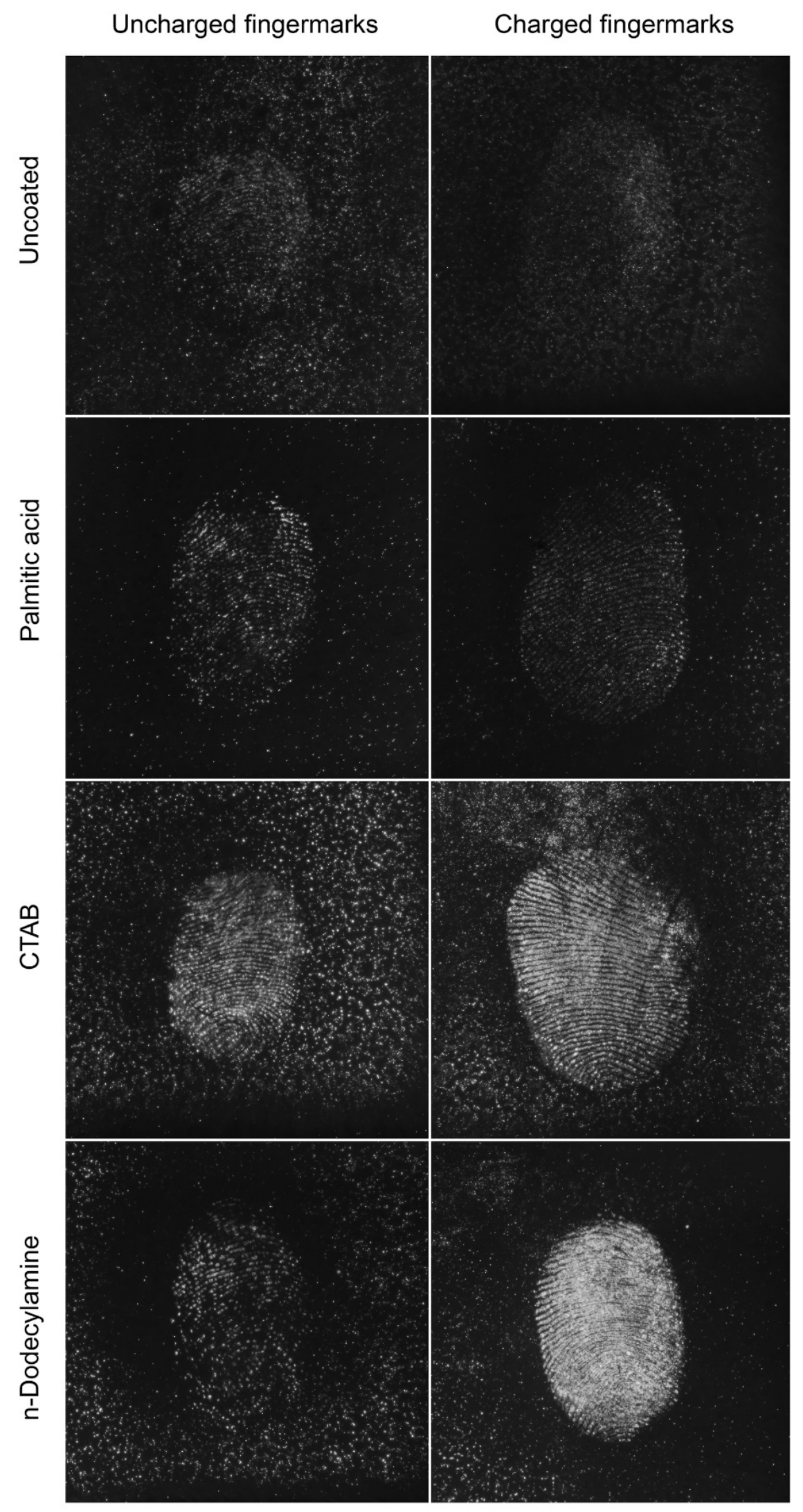

Figure 3 Photographs of developed latent fingermarks on ceramic tiles with uncoated Egyptian Blue, compared to powders coated with PA, CTAB and n-DA, the dusted fingermarks were photographed with a Canon D40 camera (illumination by a white LED array and viewed through an IR long-pass filter, aperture f/11, shutter speed 5 s, ISO 200). 

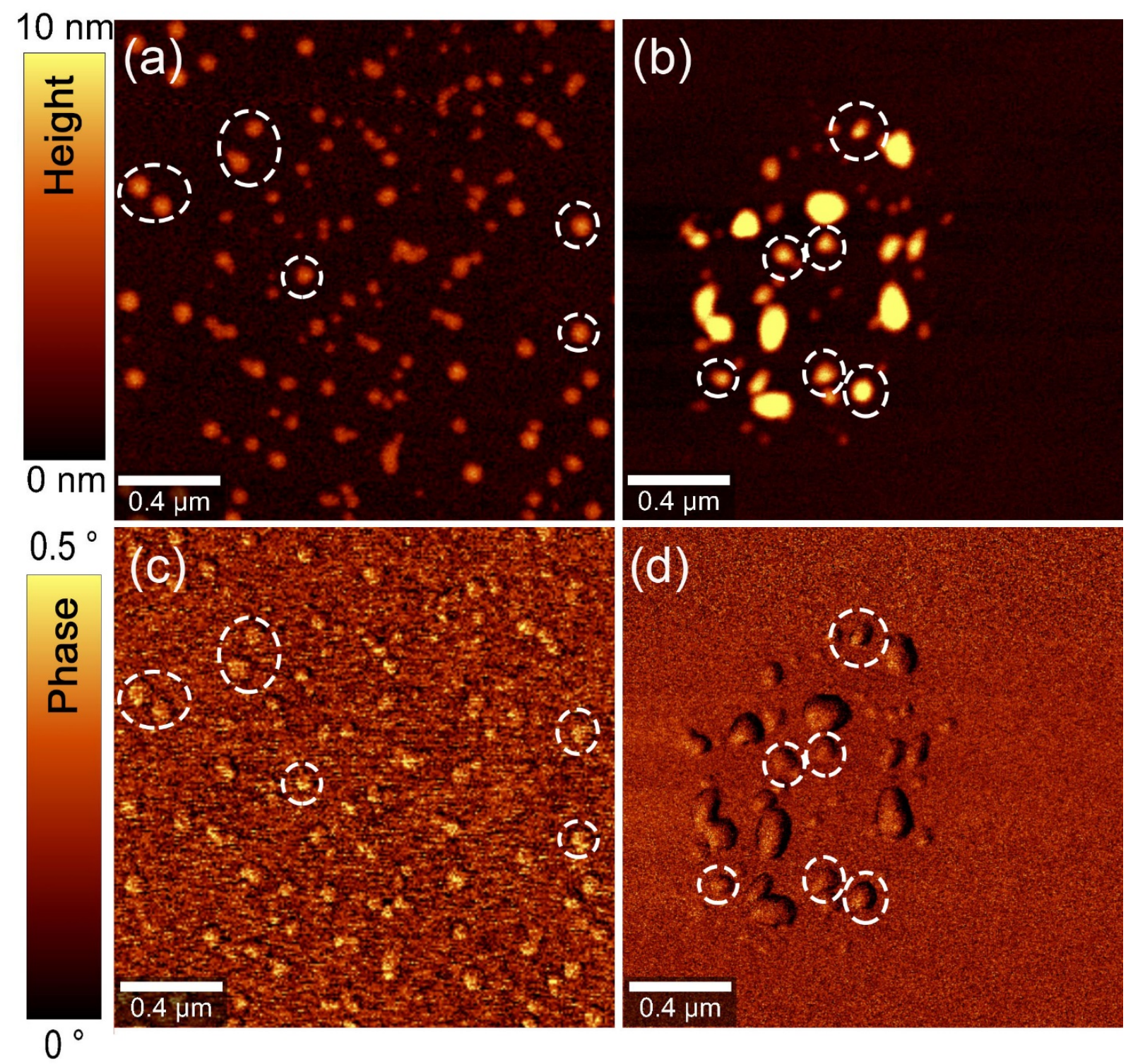

Figure 4 AFM images of topography of (a) uncoated and (b) CTAB coated exfoliated Egyptian blue nanosheets; AFM images of phase signal of (c) uncoated and (d) CTAB coated exfoliated Egyptian blue nanosheets. Dashed circles indicate coated and uncoated particles with approximately the same size.

To confirm the presence of the CTAB surfactant coating on the purified powder, FTIR was used to analyse the chemical bonding within the prepared powders. Figure 5 illustrates spectra of CTAB coated and uncoated exfoliated pigments as well as pure CTAB powder. Peaks between 420 and $1230 \mathrm{~cm}^{-1}$ have been reported in several studies for Egyptian blue powder [32-35]. These peaks can be assigned to symmetrical and antisymmetrical Si-O-Si stretching as well as $\mathrm{O}-\mathrm{Si}-\mathrm{O}$ bending [32]. The pure CTAB presented characteristic peaks at $2848 \mathrm{~cm}^{-1}$ and $2918 \mathrm{~cm}^{-1}$ that are assigned to $-\mathrm{C}-\mathrm{H}$ stretching and 
anti-stretching vibrations of the long alkyl chain of CTAB (Figure 5a) [ $\underline{36}, \underline{37}]$. Figure 5-b shows that these peaks appeared in CTAB coated particles as well; however, they were shifted to $2853 \mathrm{~cm}^{-1}$ and $2925 \mathrm{~cm}^{-1}$ respectively indicating the possible interaction between CTAB and Egyptian blue particles [료. Additional peaks in the $700-750 \mathrm{~cm}^{-1}$ and 1350-1500 $\mathrm{cm}^{-1}$ regions (Figure 5c and 5d) arising from $\mathrm{CH}_{2}$ rocking and stretching vibrations of the ammonium group $\left(\mathrm{CH}_{3}-\mathrm{N}^{+}\right)$of $\mathrm{CTAB}$ were shifted or disappeared in coated particles $[\underline{38}, \underline{39}]$. Taken together, these spectroscopic data confirm the presence of CTAB on the surface of the prepared exfoliated Egyptian blue powders.

The optical properties of the coated powders were investigated using luminescence spectroscopy. Emission spectra of the Egyptian blue, CTAB coated, and uncoated exfoliated powders are given in Figure 6. The wavelength of maximum emission for all samples is approximately $910 \mathrm{~nm}$, in the NIR region and is consistent with previous studies $[\underline{14}, \underline{18}, \underline{19}]$. As shown by the spectra, exfoliation and surfactant coating the pigment did not affect its emission characteristics. For comparison, the emission peak of fpNatural1, a commercial NIR luminescent fingermark powder, was studied as well (Figure 6). The emission peak for fpNatural1 when excited at the recommended wavelength of $580 \mathrm{~nm}$ is just outside the visible region at $730 \mathrm{~nm}$. The longer wavelength NIR emission peak from the Egyptian blue powder, combined with its much stronger luminescence quantum yield [40], leads to higher contrast images of dusted fingermarks compared to fpNatural1. 

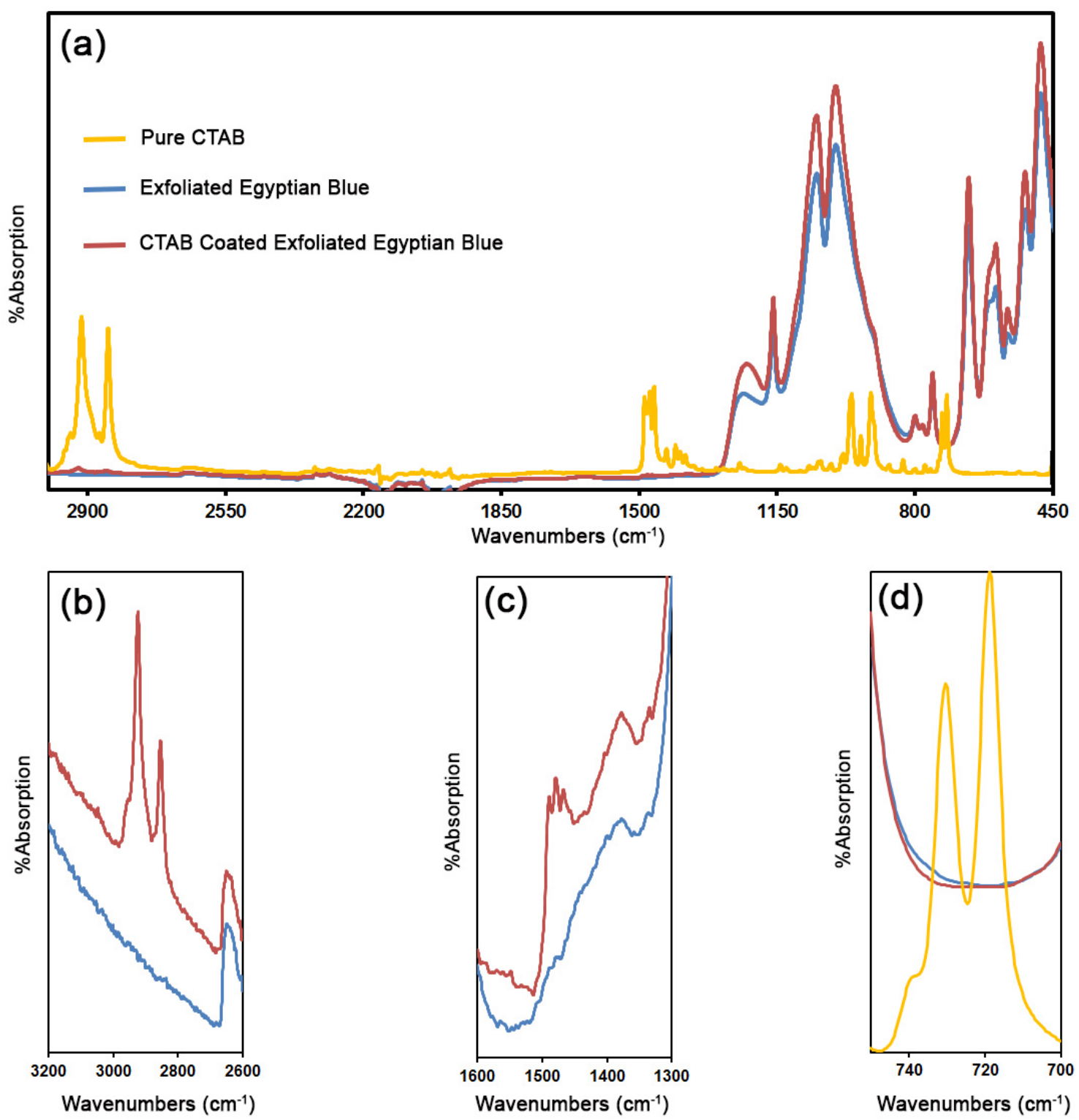

Figure 5 (a) FTIR spectra of pure CTAB powder, exfoliated Egyptian blue particles and CTAB coated exfoliated Egyptian blue particles. (b, $c$ and $d$ ) FTIR spectra in narrower ranges of wavenumbers 


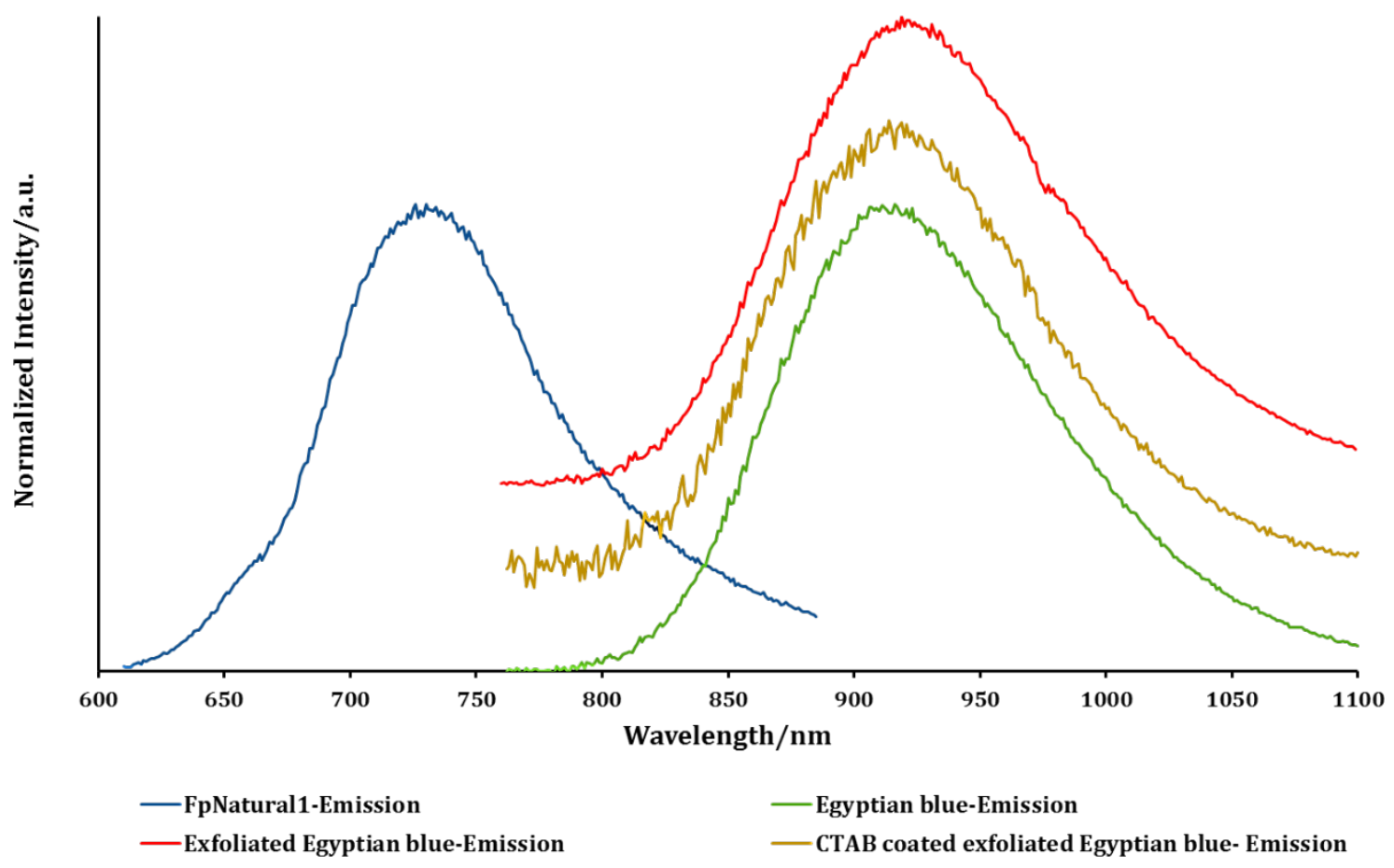

Figure 6 Luminescence emission spectra of Egyptian blue (excited at $630 \mathrm{~nm}$ ), exfoliated Egyptian blue (excited at $650 \mathrm{~nm}$ ) and CTAB coated Egyptian blue (excited at $616 \mathrm{~nm}$ ) powders as well as commercial NIR fpNatural1 pigment (excited at $580 \mathrm{~nm}$ ). Emission spectra were normalized to unity. Coated and uncoated exfoliated Egyptian blue spectra have been offset for clarity.

\subsection{Latent fingermark development}

The sensitivity of CTAB coated exfoliated Egyptian blue was compared with the micronised Egyptian blue used in previous studies [14]. Uncharged split latent fingermarks of 6 donors ( 3 males and 3 females) in depletion series were collected on glass slides and left under ambient conditions for 2 days $(3$ middle fingers of the left hand) and 15 days ( 3 middle fingers of right hand). Although the contrast between the developed latent fingermarks and the substrate was almost the same for both powders, especially for the first depletions, the difference between adherence of coated exfoliated particles and micronised ones became more noticeable in the fourth depletions of the 15-day aged latent fingermarks (Figure 7). These fingermarks should contain far less 
moisture and oily components in the fourth depletion compared to first one. With aging, the fingermarks rapidly lose moisture through evaporation, and their lipid components may change to harder, less sticky waxes because of chemical changes. In this case, an increase in the lipophilic properties of the particles by coating them with CTAB caused more lipophilic interactions, more adhesion to fingermarks, and hence better sensitivity.

To compare the sensitivity of coated and uncoated exfoliated Egyptian blue powders, $4^{\text {th }}$ depletion of 2 day-old uncharged latent fingermarks from 6 donors $(3$ males and 3 females) were powder-dusted with exfoliated Egyptian blue with and without a CTAB coating. Figure 8 shows that the fingermarks dusted with the exfoliated Egyptian blue having a lipophilic coating revealed better contrast and less background interference than similar fingermarks dusted with an uncoated Egyptian blue powder. The improvement shown by the coated particles is likely due to the lipophilic interaction between the hydrocarbon chain of CTAB and the oily (waxes, triglycerides, squalene) components and moisture within the fingermark secretions. 


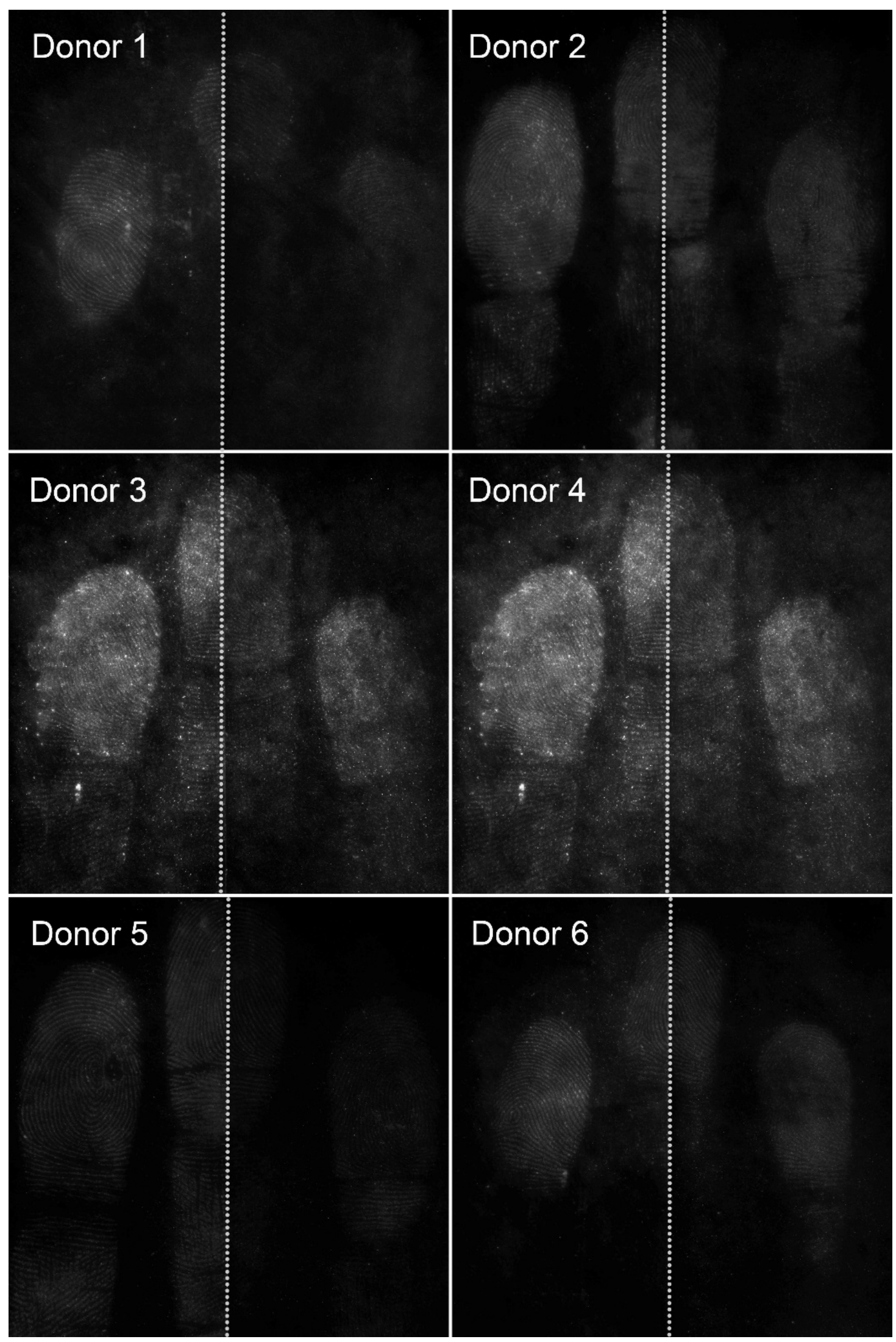

Figure 7 Photographs of developed $4^{\text {th }}$ depletion of 15-day aged split latent fingermarks of 6 donors on glass slides with (left) CTAB coated exfoliated Egyptian blue and (right) micronised Egyptian blue, photographed with Canon camera (excitation with a Polilight PL 500 at $590 \mathrm{~nm}$ excitation and viewed through an IR longpass filter, aperture f/9, shutter speed 5 s, ISO 200). 


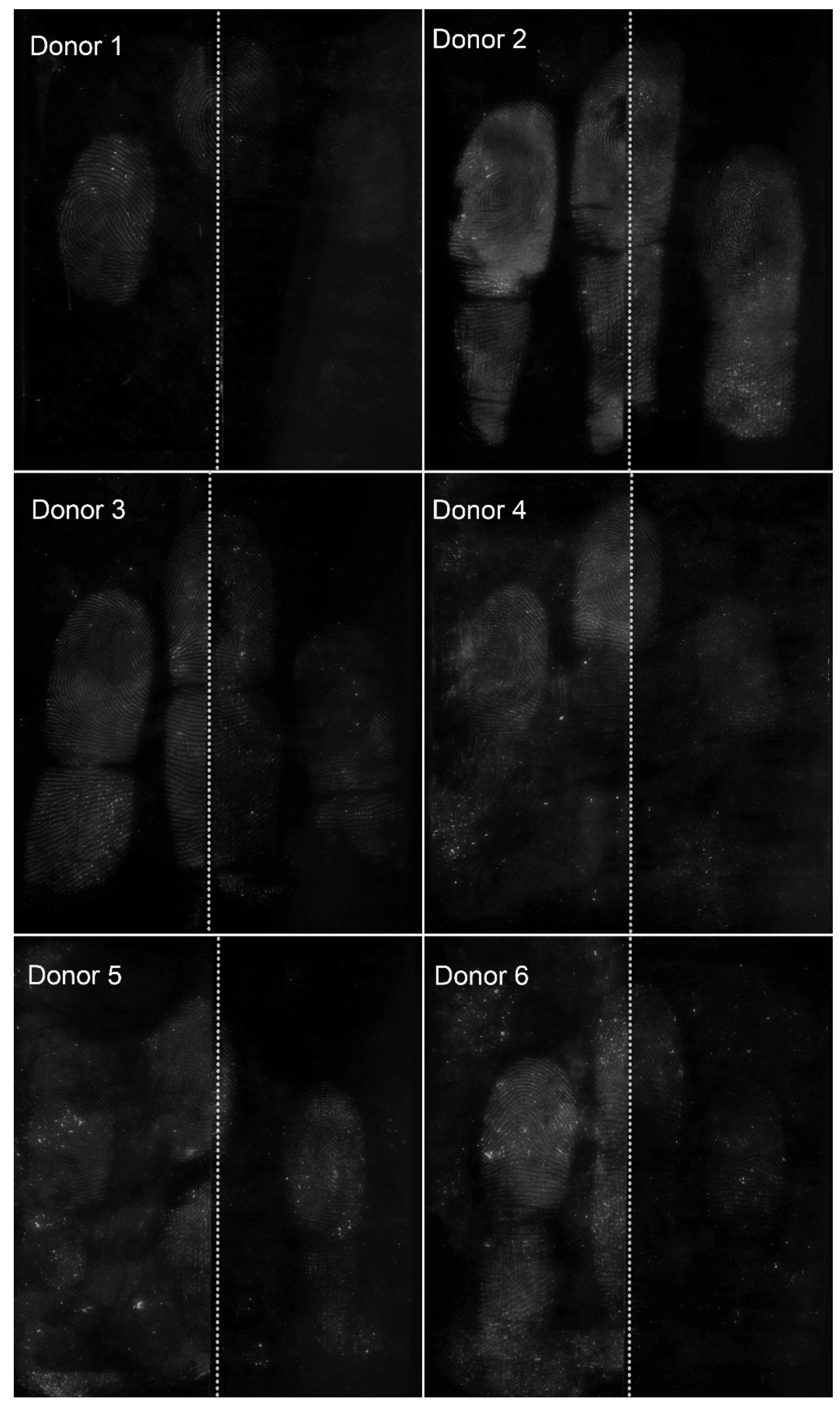

Figure 8 Photographs of developed $4^{\text {th }}$ depletions of 2-days old split latent fingermarks of 6 donors on glass slides with (left) CTAB coated exfoliated Egyptian blue and (right) exfoliated Egyptian blue, photographed with Canon camera (excitation with a Polilight PL 500 at $590 \mathrm{~nm}$ excitation and viewed through an IR longpass filter, aperture f/11, shutter speed 5 s, ISO 200). 
To demonstrate the advantages of a NIR luminescent powder for fingermark detection on multicoloured, highly patterened substrates, latent fingermark samples were collected on new 5 AUD banknotes. Figure 9 shows developed uncharged 15-day old latent fingermarks with CTAB coated exfoliated Egyptian blue. Latent fingermarks were placed on different parts of the banknote with various surface properties including the rolling colour bird (Figure 9a), transparent line (Figure 9b), the number 5 (Figure 9c), and Queen's portrait (Figure 9d). No background interference was observed in the NIR region under luminescence conditions, and the fingermark ridge details are clearly imaged.
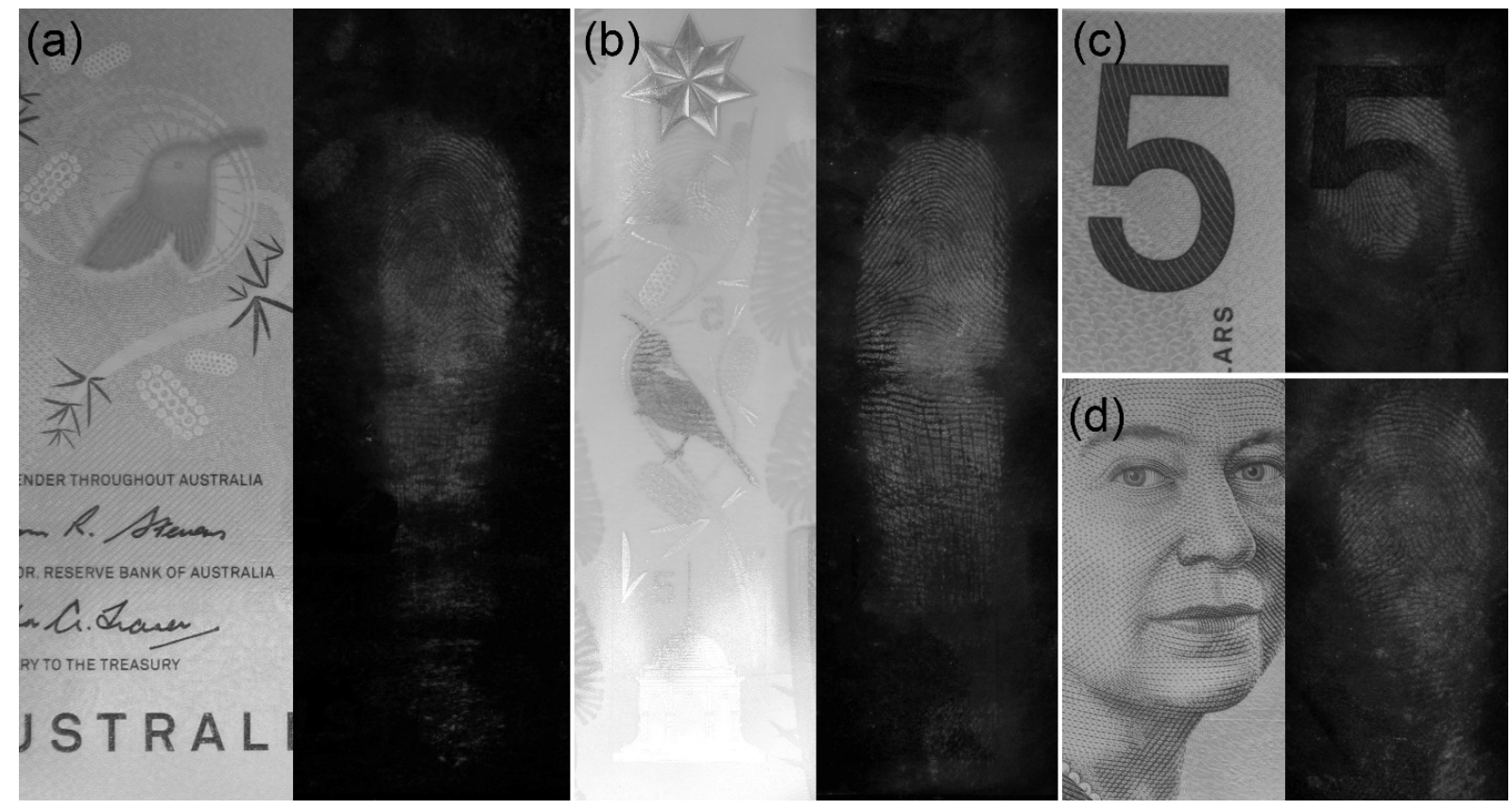

Figure 9 Photographs of latent fingermarks on a new 5 AUD banknote in the (a) rolling colour bird, (b) transparent line, (c) number 5 regions, and (d) Queen's portrait, dusted with CTAB coated exfoliated Egyptian blue and photographed with Canon camera (illumination by a white LED array and viewed through an IR long-pass filter, aperture f/9, shutter speed 8 s, ISO 200). Not the same 5 AUD note in visible and dusted were provided to show just general location rather than exact location.

Another experiment was conducted to investigate the capability of the CTAB coated exfoliate Egyptian blue powder in developing 2 month-old uncharged fingermarks on different substrates including a glass petri dish (Figure 11a and Figure 11d), a white tile (Figure 11b and Figure 11e), a ceramic tile (Figure 11c and Figure 11f) and a new 5 AUD 
banknote (Figure 11). According to Figure 10d, after 2 months, dusting fingermarks on the petri dish presented ridges in high detail. However, fingermark ridges on the white tile are not completely detectable at some regions (Figure 10e). This could be as a result of spreading the secretion through the space between the ridges due to either firmly pressing of the fingers on the substrate or fingermark ridge drift over time which has been reported before $[\underline{41}, \underline{42}]$. Ridge details of the dusted fingermarks on the ceramic tile was not developed thoroughly, however, still some ridge details are detectable (Figure 10f).

As shown in Figure 11, fingermarks were developed with diverse qualities at the different areas of the banknote; however, they were deposited from the same secretion. Developed fingermarks presented different quality in terms of ridge details. This difference could result from different surface roughness, porosity, or other surface characteristics of the mentioned areas, which needs to be studied more accurately in the future. Consequently, results illustrated in Figure 10 and Figure 11 suggest a high sensitivity of the CTAB coated exfoliated Egyptian powder. 

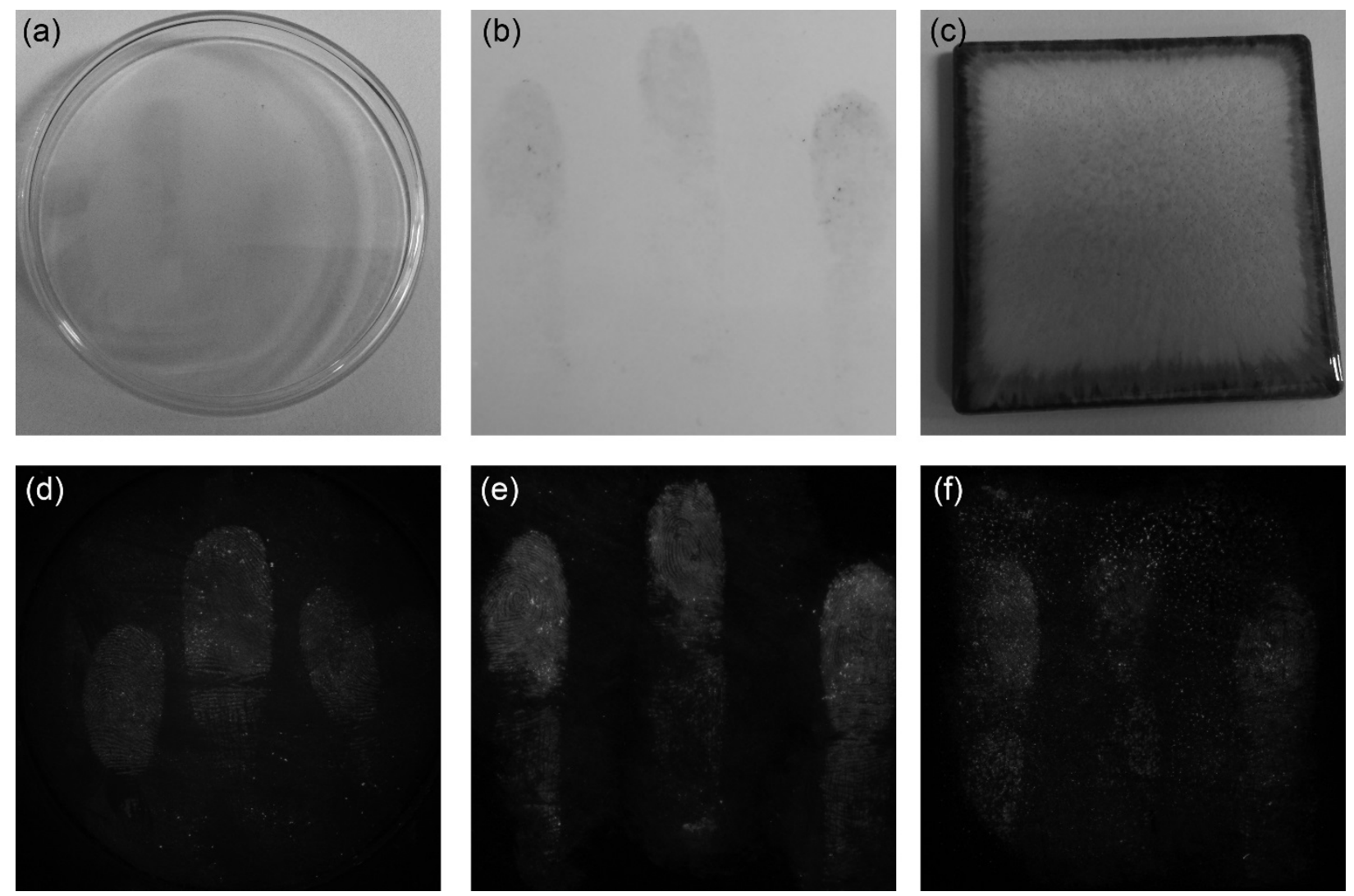

Figure 10 Photographs of visible and luminescent latent fingermarks on a (a) and (d) glass petri dish, (b) and

(e) a white tile, (c) and (f) a ceramic tile dusted with CTAB coated exfoliated Egyptian blue and photographed with Canon camera (illumination by a white LED array and viewed through an IR long-pass filter, aperture f/9, shutter speed 2 s, ISO 200). 


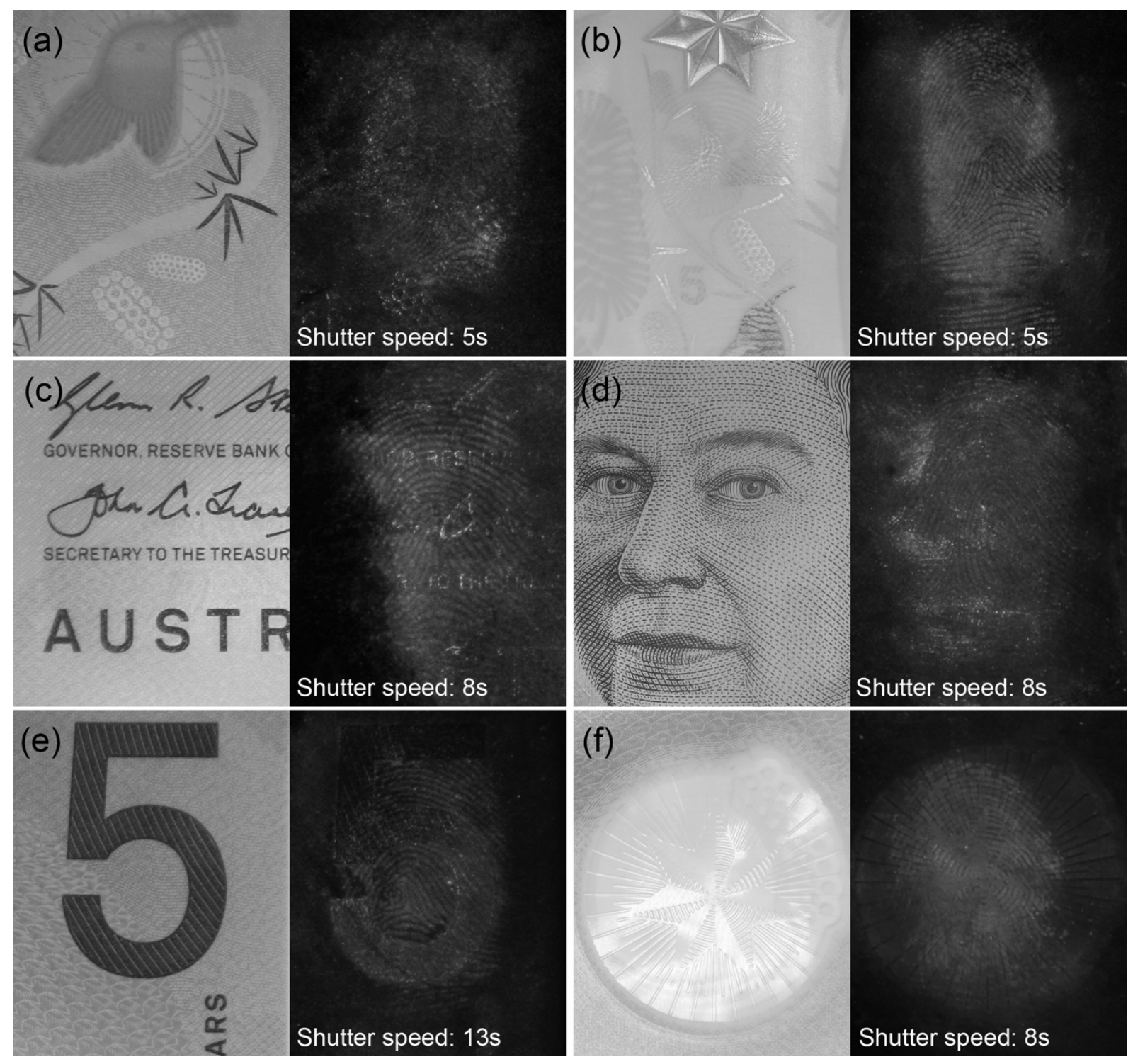

Figure 11 Photographs of latent fingermarks on a new 5 AUD banknote in the (a) rolling colour bird, (b) transparent line, (c) fluorescent ink, (d) Queen's portrait, (e) number 5 regions, and (f) clear window, dusted with CTAB coated exfoliated Egyptian blue and photographed with Canon camera (illumination by a white LED array and viewed through an IR long-pass filter, aperture f/9, ISO 200). Not the same 5 note in visible and dusted were provided to show general location rather than exact location.

Moreover, the NIR luminescent property of the powder presents more desirable results when it was compared to Blitz Red, another commercial visible luminescent fingermark powder. This was especially the case when they were used for detecting latent fingermarks on the Queen's portrait (Figure 12a). Figure 12a shows that there is a visible pattern on the Queen's face which is very similar to fingermark ridges and makes imaging latent fingermark patterns using Blitz Red challenging (Figure 12b). However, 
the background was entirely eliminated in the photograph of developed latent fingermarks dusted with CTAB coated exfoliated Egyptian blue (Figure 12c).
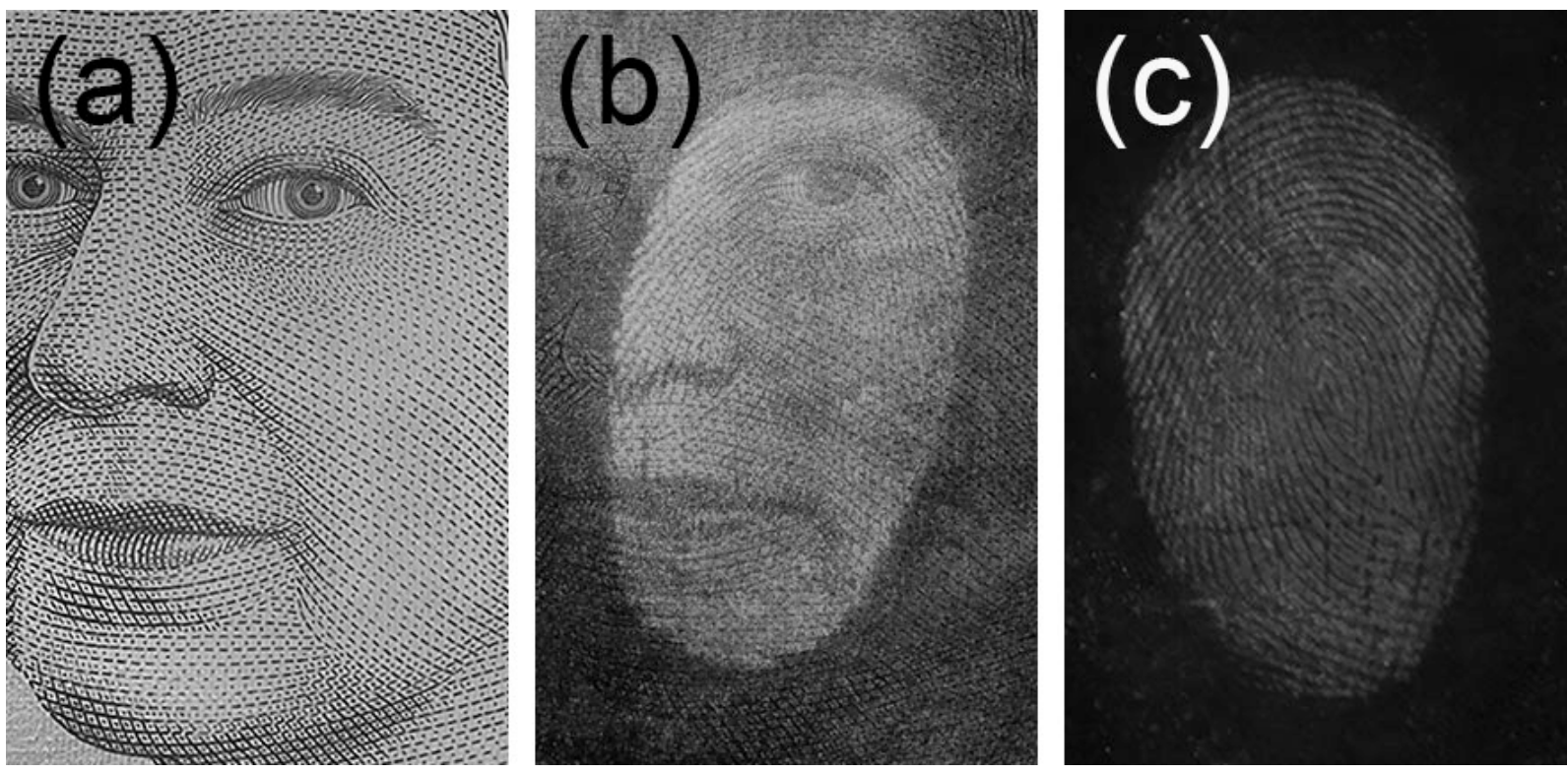

Figure 12 Photographs of (a) Queen's portrait and developed latent fingermarks on new 5 AUD banknotes with (b)Blitz Red, photographed with Nikon camera (excitation with a Polilight PL 500 at $505 \mathrm{~nm}$ excitation and viewed through an $529 \mathrm{~nm}$ orange filter, aperture f/11, shutter speed $1 \mathrm{~s}$, ISO 200); and (b) CTAB coated exfoliated Egyptian blue, photographed with Canon camera (illumination by a white LED array and viewed through an IR long-pass filter, aperture f/9, shutter speed 1 s, ISO 200).

\section{Conclusions}

In this study, NIR luminescent Egyptian blue particles were decreased in size and coated with a lipophilic surfactant, leading to a simple and inexpensive method to enhance the sensitivity of the powder for detection of latent fingermarks. Advanced methods for materials analysis successfully characterized the prepared powders. Fresh and aged latent fingermarks in depletion series were collected on glass slides as typical nonporous substrates and on new 5 AUD banknotes as examples of multicoloured and patterned substrates. These surfaces were then developed with conventional Blitz Red® florescent powder, micronised Egyptian blue, exfoliated Egyptian blue and surfacecoated exfoliated Egyptian blue. Little difference was observed in the quality of developed fresh fingermarks dusted with exfoliated Egyptian blue and micronised 
Egyptian blue powders. However, noticeable enhancement was achieved in fingermark detection when aged fingermarks, especially in their $4^{\text {th }}$ depletions, were powdered with coated exfoliated Egyptian blue rather than uncoated exfoliated and micronised developed ones. Moreover, the comparison between coated exfoliated Egyptian blue and Blitz Red ${ }^{\circledR}$ revealed better results for exfoliated Egyptian blue dusted fingermarks on patterned substrates.

\section{Acknowledgments}

The authors would like to acknowledge Dr. Mark Hackett and Dr. Amanda Frick (Curtin University) for their support during this research. The authors thank all of the fingermark donors for their cooperation. J. V. Goodpaster thanks the IUPUI School of Science and IUPUI Sabbatical Leave Committee for their support of his time at Curtin University. S. Shahbazi is supported by an Australian Postgraduate Award. The authors declare no competing financial interest. This research has been conducted in accordance with the Curtin University Human Research Ethics Committee (Approval Number HRE2016-0252).

\section{References}

1. Chadwick, S., et al., Styryl dye coated metal oxide powders for the detection of latent fingermarks on non-porous surfaces. Forensic Sci. Int., 2012. 219(1-3): p. 208-214.

2. Sharma, V., et al., Potential of Sr4Al14025: Eu2+,Dy3+ inorganic oxide-based nanophosphor in Latent fingermark detection. J. Mater. Sci., 2013. 49(5): p. 2225-2234.

3. Stoilovic, M. and C. Lennard, NCFS workshop manual: fingermark detection \& enhancement. 6th ed. 2012, Canberra: National Centre for Forensic Studies.

4. Tahtouh, M., et al., Four novel alkyl 2-cyanoacylate monomers and their use in latent fingermark detection by mid-infrared spectral imaging. Forensic Science International, 2011. 207(1-3): p. 223-238.

5. M. Tahtouh, P.D., R. Shimmon, J.R. Kalman, B.J. Reedy, The application of infrared chemical imaging to the detection and enhancement of latent fingerprints: method optimization and further findings. Journal of Forensic Sciences, 2007. 52(1089-1096.).

6. Chadwick, S., et al., Use of Styryl 11 and STaR 11 for the Luminescence Enhancement of Cyanoacrylate-Developed Fingermarks in the Visible and Near-Infrared Regions. Journal of Forensic Sciences, 2011. 56(6): p. 1505-1513.

7. Maynard, P., et al., Near infrared imaging for the improved detection of fingermarks on difficult surfaces. Australian Journal of Forensic Science, 2009. 41(Issue 1): p. 43-62. 
8. Chadwick, S., et al., Styryl dye coated metal oxide powders for the detection of latent fingermarks on non-porous surfaces. Forensic Science International, 2012. 219(1-3): p. 208-214.

9. Jin, X., et al., NIR luminescence for the detection of latent fingerprints based on ESIPT and AIE processes. RSC Advances, 2015. 5(106): p. 87306-87310.

10. King, R.S., P.M. Hallett, and D. Foster, Seeing into the infrared: a novel IR fluorescent fingerprint powder. Forensic Sci Int, 2015. 249: p. e21-6.

11. Bramble, S.K., et al., Deep red to near infrared (NIR) fluorescence of gentian violet-treated latent prints. Journal of Forensic Identification, 2000. 50(1): p. 33-49.

12. Chadwick, S., et al., Use of Styryl 11 and STaR 11 for the Luminescence Enhancement of Cyanoacrylate-Developed Fingermarks in the Visible and Near-Infrared Regions*. Forensic Sci. Int., 2011. 56(6): p. 1505-1513.

13. Maynard, P., et al., Near infrared imaging for the improved detection of fingermarks on difficult surfaces. Australian Journal of Forensic Sciences, 2009. 41(1): p. 43-62.

14. Errington, B., et al., Micronised Egyptian blue pigment: A novel near-infrared luminescent fingerprint dusting powder. Dyes and Pigments, 2016. 132: p. 310-315.

15. King, R.S.P., P.M. Hallett, and D. Foster, NIR-NIR fluorescence: A new genre of fingermark visualisation techniques. Forensic Science International, 2016. 262: p. e28-e33.

16. Pabst, A., Structures of Some Tetragonal Sheet Silicates. Acta Cryst., 1959. 12: p. 733-739.

17. Mazzi, F. and A. Pabst, Reexamination of cuprorivaite. The American Mineralogist, 1962. 47: p. 409-411.

18. Bianchetti, P., et al., Production and characterization of Egyptian blue and Egyptian green frit. Journal of Cultural Heritage, 2000. 1(2): p. 179-188.

19. Pozza, G., et al., Photoluminescence of the inorganic pigments Egyptian blue, Han blue and Han purple. Journal of Cultural Heritage, 2000. 1(4): p. 393-398.

20. Accorsi, G., et al., The exceptional near-infrared luminescence properties of cuprorivaite (Egyptian blue). Chemical Communications, 2009: p. 3392-3394.

21. Verri, G., The spatially resolved characterisation of Egyptian blue, Han blue and Han purple by photo-induced luminescence digital imaging. Analytical and Bioanalytical Chemistry, 2009. 394(4): p. 1011-1021.

22. Frey, F.S., et al., The AIC guide to digital photography and conservation documentation. 2 ed. 2011: American Institute for Conservation of Historic and Artistic Works.

23. Johnson-McDaniel, D., et al., Nanoscience of an Ancient Pigment. Journal of the American Chemical Society, 2013. 135(5): p. 1677-1679.

24. Errington, B., An alternative method for the detection of latent fingermarks using NIR luminescence imaging, in Department of Chemistry. 2013, Curtin University. p. 56.

25. Archer, N.E., et al., Changes in the lipid composition of latent fingerprint residue with time after deposition on a surface. Forensic Science International, 2005. 154(2-3): p. 224-239.

26. Frick, A.A., et al., Investigations into the initial composition of latent fingermark lipids by gas chromatography-mass spectrometry. Forensic Science International, 2015. 254: p. 133-147.

27. Huang, W., et al., Synthesis of amphiphilic silica nanoparticles for latent fingerprint detection. Analytical Letters, 2014. 48(9): p. 1524-1535.

28. Sodhi, G. and J. Kaur, A novel, nanoparticle-size fingerprint dusting composition based on eosin $B$ stain. The Indian Police Journal, 2008: p. 46.

29. IFRG, Guidelines for the assessment of fingermark detection techniques. J. Forensic Identif., 2014. 64(2): p. 174-200.

30. Frey, F. and D. Heller, The AIC guide to digital photography and conservation documentation. 2008.

31. Raghavan, D., et al., Mapping polymer heterogeneity using atomic force microscopy phase imaging and nanoscale indentation. Macromolecules, 2000. 33(7): p. 2573-2583.

32. Lluveras, A., et al., Evidence for the use of egyptian blue in an 11th century mural altarprice by SEMEDS, FTIR and SRXRD (church of Sant Pere, Terrassa, Spain). Archaeometry, 2010. 52(2): p. 308319.

33. Mirti, P., et al., Spectrochemical and structural studies on a roman sample of Egyptian blue. Spectrochimica Acta Part A: Molecular and Biomolecular Spectroscopy, 1995. 51(3): p. 437-446.

34. Mahmoud, H.M. and L. Papadopoulou, Archaeometric Analysis of Pigments from the Tomb of NakhtDjehuty (TT189), El-Qurna Necropolis, Upper Egypt. ArchéoSciences, 2013(1): p. 19-33.

35. Bruni, S., et al., Spectrochemical characterization by micro-FTIR spectroscopy of blue pigments in different polychrome works of art. Vibrational Spectroscopy, 1999. 20(1): p. 15-25.

36. Lu, C., et al., Carboxyl-polyethylene glycol-phosphoric acid: a ligand for highly stabilized iron oxide nanoparticles. Journal of Materials Chemistry, 2012. 22(37): p. 19806-19811. 
37. Barhoum, A., et al., Effect of cationic and anionic surfactants on the application of calcium carbonate nanoparticles in paper coating. ACS Applied Materials \& Interfaces, 2014. 6(4): p. 27342744.

38. Nithiyanantham, U., et al., Low temperature formation of rectangular PbTe nanocrystals and their thermoelectric properties. New Journal of Chemistry, 2016. 40(1): p. 265-277.

39. Jiang, Z., et al., Modifiers-assisted formation of nickel nanoparticles and their catalytic application to p-nitrophenol reduction. CrystEngComm, 2013. 15(3): p. 560-569.

40. Accorsi, G., et al., The exceptional near-infrared luminescence properties of cuprorivaite (Egyptian blue). Chemical Communications, 2009(23): p. 3392-3394.

41. De Alcaraz-Fossoul, J., et al., Fingermark ridge drift. Forensic Sci Int, 2016. 258: p. 26-31.

42. Dorakumbura, B.N., T. Becker, and S.W. Lewis, Nanomechanical mapping of latent fingermarks: A preliminary investigation into the changes in surface interactions and topography over time. Forensic Science International, 2016. 267: p. 16-24. 